\title{
Markov Property
}

Recent developments on the quantum Markov property

Work partially supported by INTAS n. 991/545

\section{Luigi Accardi}

Centro Interdisciplinare Vito Volterra

II Università di Roma "Tor Vergata"

Via di Tor Vergata, 00133 Roma, Italy

accardi@@volterra.uniroma2.it

\section{Francesco Fidaleo}

Dipartimento di Matematica and Centro Interdisciplinare Vito Volterra

Università di Roma (Tor Vergata)

Via della Ricerca Scientifica, 00133 Roma, Italy

fidaleo@@mat.uniroma2.it 


\section{Indice}

1 Introduction 3

2 The quantum Markov property on the spin algebra 5

3 The relation with statistical mechanics $\quad 9$

4 Markov states on non homogeneous chains: general properties 11

5 The structure of Markov states on chains: a reconstuction theorem 14

6 Quantum Markov states on general quasi-local algebras 19

7 Acknowledgements 20 


\begin{abstract}
We review recent developments in the theory of quantum Markov states on the standard $\mathbb{Z}^{d}$-spin lattice. A Dobrushin theory for quantum Markov fields is proposed. In the one-dimensional case where the order plays a crucial role, the structure arising from a quantum Markov state is fully understood. In this situation we obtain a splitting of a Markov state into a classical part, and a purely quantum part. This result allows us to provide a reconstruction theorem for quantum Markov states on chains.
\end{abstract}

Mathematics Subject Classification: 46L53, 46L60, 60J99, 82B10. Key words: Non commutative measure, integration and probability; Quantum Markov processes; Mathematical quantum statistical mechanics.

\title{
1 Introduction
}

The problem of introducing a notion of quantum Markov field, explicit enough to allow a quantum generalization of Dobrushin's theory, has been open for several years. Recent advances in the structure theory of Markov states on chains $([3,6])$ have suggested a natural multi-dimensional generalization of the notion of Markov state, see [4]. Such a notion has the advantage of being entirely expressible in terms of Umegaki conditional expectations with additional localization properties. This allows to formulate a quantum Dobrushin theory for Markov fields which exactly parallels the classical theory, at the basis of equilibrium statistical mechanics.

In the present paper we review recent developments in the theory of quantum Markov states on the standard $\mathbb{Z}^{d}$-spin lattice.

In the one-dimensional case where the order plays a crucial role, the structure arising from a quantum Markov state is fully understood. Following previous results of $[5,6]$, a splitting of a Markov state into a classical part, and a purely quantum part was obtained in [3]. This result allowed us to provide a reconstruction theorem for quantum Markov states on chains. Further, it emerged that the Markov property for a locally faithful state $\varphi$ on the spin algebra $\mathfrak{A}$ on the chain, can be equivalently established through properties of generalized conditional expectations defined in [2], which are canonical objects intrinsically associated to the local structure of the quasilocal algebra $\mathfrak{A}$, and the state $\varphi$ under consideration. This was done by discovering the existence of a very explicit nearest neighbour Hamiltonian canonically associated to the Markov state $\varphi$, which generates on the quasi- 
local algebra $\mathfrak{A}$, a one-parameter group of automorphisms admitting $\varphi$ as a KMS-state.

Taking into account the suggestion emerging from one-dimensional models, the intrinsic definition of the Markov property in terms of properties of generalized conditional expectations, was the starting-point in [4], in order to investigate the general multi-dimensional case. For these quantum Markov fields (i.e. quantum Markov processes with multi-dimensional indices), deep connections with the KMS boundary condition, as well as phenomena of phase transitions and symmetry breaking, naturally emerge, generalizing the classical situation, see $[10,11,12]$.

Every quantum Markov field is canonically associated to a (non-commutative) potential. The problem to give a full reconstruction theorem for these potentials remains still open. However, the conditions on the potential associated to a Markov state, could be explicit enough to allow the construction of a multiplicity of non trivial examples.

We conclude the introduction by recalling some standard definitions used in the sequel.

We consider quasi-local algebras obtained in the following way. For each $j$ in an index set $I$, a finite-dimensional $C^{*}$-algebra $M^{j}$ is assigned and, for each finite subset $\Lambda \subset I$, we define

$$
\mathfrak{A}_{\Lambda}:=\otimes_{j \in \Lambda} M^{j}
$$

The quasi-local algebra $\mathfrak{A}$ is the $C^{*}$-inductive limit associated to the directed system $\left\{\mathfrak{A}_{\Lambda}\right\}_{\Lambda \in I}$ with the natural embeddings

$$
\iota_{\Lambda, \widehat{\Lambda}}: A_{\Lambda} \in \mathfrak{A}_{\Lambda} \rightarrow A_{\Lambda} \otimes I_{\widehat{\Lambda} \backslash \Lambda} \in \mathfrak{A}_{\widehat{\Lambda}}, \quad \Lambda \subset \widehat{\Lambda}
$$

In this situation we write

$$
\mathfrak{A}:=\otimes_{j \in I} M^{j}
$$

where the infinite tensor product is defined w.r.t. the unique $C^{*}$-cross norm. We often denote by $\iota_{\Lambda}: \mathfrak{A}_{\Lambda} \mapsto \mathfrak{A}$ the canonical injection of $\mathfrak{A}_{\Lambda}$ into $\mathfrak{A}$ and refer to [8] for further details.

We associate a fixed bounded "boundary" $\partial \Lambda \subset \Lambda^{\prime}$ to each bounded region $\Lambda$, where $\Lambda^{\prime}$ is the complement of $\Lambda$. In such a situation, we define

$$
\bar{\Lambda}:=\Lambda \cup \partial \Lambda
$$


and write $\Lambda \subset \subset \widehat{\Lambda}$ if $\bar{\Lambda} \subset \widehat{\Lambda}$.

By a (Umegaki) conditional expectation $E: \mathfrak{A} \mapsto \mathfrak{B} \subset \mathfrak{A}$ we mean a norm-one projection of the $C^{*}$-algebra $\mathfrak{A}$ onto a $C^{*}$-subalgebra $\mathfrak{B}$ with the same identity. The map $E$ is automatically a completely positive identitypreserving $\mathfrak{B}$-bimodule map, see [20], Section 9 . When $\mathfrak{A}$ is a matrix algebra, the structure of a conditional expectation is well-known, see [14], Proposition 2.2 , together with [6], Lemma 3.1. Namely, suppose that $\mathfrak{A}$ is a full matrix algebra and consider the (finite) set $\left\{P_{i}\right\}$ of minimal central projections of the range $\mathfrak{B}$ of $E$, we have

$$
E(x)=\sum_{i} E\left(P_{i} x P_{i}\right) P_{i}
$$

Then $E$ is uniquely determined by its values on the reduced algebras

$$
\mathfrak{A}_{P_{i}}:=P_{i} \mathfrak{A} P_{i}=N_{i} \otimes \bar{N}_{i}
$$

where $N_{i} \sim \mathfrak{B} P_{i}$ and $\bar{N}_{i} \sim P_{i}\left(\mathfrak{B}^{\prime} \wedge \mathfrak{A}\right) P_{i}$. In fact, there exist states $\phi_{i}$ on $\bar{N}_{i}$ such that

$$
E\left(P_{i}(a \otimes \bar{a}) P_{i}\right)=\phi_{i}(\bar{a}) P_{i}(a \otimes I) P_{i} .
$$

Let $N \subset M$ be an inclusion of von Neumann algebras equipped with a normal faithful state on $M$. By the $\varphi$-conditional expectation we mean the identity-preserving normal faithful completely positive map

$$
E^{\varphi}: M \mapsto N
$$

defined in [2]. Such a map preserves the state $\varphi$ and seems to be the natural generalization of the concept of conditional expectation in the sense of Umegaki, even if the former is not in general a norm-one projection.

Such a $\varphi$-conditional expectation is written as

$$
\pi_{\varphi_{\Gamma N}}\left(E^{\varphi}(a)\right)=J_{\varphi_{\Gamma N}} P_{\varphi_{\Gamma N}} J_{\varphi} \pi_{\varphi}(a) J_{\varphi} J_{\varphi_{\Gamma N}}
$$

where the $J$ are the Tomita antiunitary conjugations, and $P_{\varphi_{\Gamma N}} \in \pi_{\varphi}(N)^{\prime}$ is the cyclic projection relative to $\pi_{\varphi}(N)$.

\section{The quantum Markov property on the spin algebra}

The investigation of the quantum Markov property relies on the concept of generalized conditional expectation (denoted also by $\varphi$-conditional expectation when the state $\varphi$ is specified) firstly introduced in [2]. 
Let $\mathfrak{A}$ be the spin algebra on $\mathbb{Z}^{d}$ given by

$$
\mathfrak{A}:=\otimes_{i \in \mathbb{Z}^{d}} M^{i}
$$

where $M^{i}=\mathbb{M}_{k}(\mathbb{C})$, a fixed full matrix algebra. Suppose we have a locally faithful state $\varphi$ on $\mathfrak{A}$ with restrictions $\varphi_{\Lambda}, \Lambda \subset \mathbb{Z}^{d}$ bounded, to local algebras $\mathfrak{A}_{\Lambda}$. Consider a pair $\Lambda \subset \subset \widehat{\Lambda}$ of bounded regions of $\mathbb{Z}^{d}$. Denote

$$
E_{\widehat{\Lambda} \backslash \Lambda}^{\varphi_{\widehat{\Lambda}}}: \mathfrak{A}_{\widehat{\Lambda}} \mapsto \mathfrak{A}_{\widehat{\Lambda} \backslash \Lambda}
$$

the $\varphi_{\widehat{\Lambda}}$-conditional expectation relative to the inclusion $\mathfrak{A}_{\widehat{\Lambda} \backslash \Lambda} \subset \mathfrak{A}_{\widehat{\Lambda}}$.

Definition 2.1 The locally faithful state $\varphi \in \mathcal{S}(\mathfrak{A})$ is said to be a Markov state if for every pair of regions $\Lambda, \widehat{\Lambda}$ as above, we have

$$
\mathfrak{A}_{\widehat{\Lambda} \backslash \bar{\Lambda}} \subset \operatorname{Fix}\left(E_{\widehat{\Lambda} \backslash \Lambda}^{\varphi_{\widehat{\Lambda}}}\right)
$$

Condition (2) simply means that $E_{\widehat{\Lambda} \backslash \Lambda}^{\varphi_{\widehat{\Lambda}}}$ acts trivially on the observables localized in $\widehat{\Lambda} \backslash \bar{\Lambda}$.

It is of interest to formulate the quantum Markov property in terms of a net of Umegaki conditional expectations which leave the state $\varphi$ invariant. This can be easily done for each finite-volume theory.

Namely, fix a large but bounded region $\widehat{\Lambda}$ and consider all the bounded regions $\Lambda$ with $\Lambda \subset \subset \widehat{\Lambda}$. Then, taking the ergodic averages of the $\varphi_{\widehat{\Lambda}^{-}}$ conditional expectations (see [15] for the most general situation), we obtain a set $\left\{E_{\widehat{\Lambda} \backslash \Lambda}\right\}_{\Lambda \subset \subset \widehat{\Lambda}}$ of Umegaki conditional expectations as

$$
E_{\widehat{\Lambda} \backslash \Lambda}:=\lim _{n} \frac{1}{n} \sum_{k=0}^{n-1}\left(E_{\widehat{\Lambda} \backslash \Lambda}^{\varphi_{\widehat{\Lambda}}}\right)^{k}
$$

By Theorem 5.1 of [2], such conditional expectations project onto the largest expected subalgebras of $\mathfrak{A}_{\widehat{\Lambda} \backslash \Lambda}$, and leave invariant by constuction the state $\varphi$. Moreover, by the Markov property (2), the $E_{\widehat{\Lambda} \backslash \Lambda}$ factorize as

$$
E_{\widehat{\Lambda} \backslash \Lambda}=\mathcal{E}_{\widehat{\Lambda} \backslash \Lambda} \otimes \operatorname{id}_{\mathfrak{A}_{\widehat{\Lambda} \backslash \Lambda}}
$$

where $\mathcal{E}_{\widehat{\Lambda} \backslash \Lambda}: \mathfrak{A}_{\widehat{\Lambda}} \mapsto \mathfrak{B}_{\partial \Lambda}^{\widehat{\Lambda}} \subset \mathfrak{A}_{\partial \Lambda}$ is given by

$$
\mathcal{E}_{\widehat{\Lambda} \backslash \Lambda}:=E_{\widehat{\Lambda} \backslash \Lambda}\left\lceil\mathfrak{A}_{\bar{\Lambda}}\right.
$$

In order to understand the infinite-volume theory, one should investigate the behavior of $\mathfrak{B}_{\partial \Lambda}^{\widehat{\Lambda}} \equiv \mathcal{R}\left(\mathcal{E}_{\widehat{\Lambda} \backslash \Lambda}\right)$ as $\widehat{\Lambda}$ increases in order to exhaust oll of $\mathbb{Z}^{d}$. 
Theorem 1 (Theorem 3.3 of [4])

Let $\varphi \in \mathcal{S}(\mathfrak{A})$ be a Markov state.

For each bounded region $\Lambda \subset \mathbb{Z}^{d}$ there exists a Umegaki conditional expectation $E_{\Lambda^{\prime}}$ on $\mathfrak{A}$, which projects into $\mathfrak{A}_{\Lambda^{\prime}}$.

Moreover, the net $\left\{E_{\Lambda^{\prime}}\right\}_{\Lambda \subset \mathbb{Z}^{d}}$ satisfies:

(i) $E_{\Lambda^{\prime}}\left(\mathfrak{A}_{\bar{\Lambda}}\right) \subset \mathfrak{A}_{\partial \Lambda}$,

(ii) $E_{\Lambda^{\prime}}\left\lceil_{\mathfrak{A}_{\bar{\Lambda}^{\prime}}}=\operatorname{id}_{\mathfrak{A}_{\bar{\Lambda}^{\prime}}}\right.$,

(iii) $\varphi \circ E_{\Lambda^{\prime}}=\varphi$,

(iv) if $\Lambda_{1} \subset \Lambda_{2}$ then

$$
E_{\Lambda_{2}^{\prime}} E_{\Lambda_{1}^{\prime}}=E_{\Lambda_{2}^{\prime}}
$$

Proof 1 We report only a sketch of the proof given in [4]. By Theorem 5.1 of [2], it follows that the ranges $\mathfrak{B}_{\partial \Lambda}^{\widehat{\Lambda}}$ of the $\mathcal{E}_{\widehat{\Lambda} \backslash \Lambda}$ give rise to a decreasing net of subalgebras of $\mathfrak{A}_{\partial \Lambda}$, taking into account (4).

Namely, $\left\{\mathcal{E}_{\widehat{\Lambda} \backslash \Lambda}\right\}_{\widehat{\Lambda} \supset \supset \Lambda}$ is a decreasing net of conditional expectations defined on the full matrix algebra $\mathfrak{A}_{\bar{\Lambda}}$, which converges to a conditional expectation by a standard martingale convergence theorem, see [22], Theorem 3.

Denoting

$$
\mathcal{E}_{\Lambda^{\prime}}:=\lim _{\widehat{\Lambda} \uparrow \mathbb{Z}^{d}} \mathcal{E}_{\widehat{\Lambda} \backslash \Lambda}
$$

the expectation $\mathcal{E}_{\Lambda^{\prime}}$ projects onto ${ }^{1}$

$$
\mathfrak{B}_{\partial \Lambda}:=\bigcap_{\widehat{\Lambda} \subset \mathbb{Z}^{d}} \mathfrak{B}_{\partial \Lambda}^{\hat{\Lambda}} \subset \mathfrak{A}_{\partial \Lambda}
$$

and gives rise to the searched conditional expectation

$$
E_{\Lambda^{\prime}}:=\mathcal{E}_{\Lambda^{\prime}} \otimes \operatorname{id}_{\mathfrak{A}_{\bar{\Lambda}^{\prime}}}
$$

The net $\left\{E_{\Lambda^{\prime}}\right\}_{\Lambda \subset \mathbb{Z}^{d}}$ is projective, and leaves by construction the state $\varphi$ invariant.

\footnotetext{
${ }^{1}$ The Umegaki conditional expectations $\left\{\mathcal{E}_{\Lambda^{\prime}}\right\}$ are called sometimes transition expectations.
} 
In order to investigate the converse of Theorem 1, we consider the potential associated to a locally faithful state $\varphi$ consistent with a net $\left\{E_{\Lambda^{\prime}}\right\}$ of conditional expectations as that in Theorem 1.

Suppose we have a locally faithful state on the quasi-local algebra $\mathfrak{A}$, then a selfadjoint operator $h_{\Lambda} \in \mathfrak{A}_{\Lambda}$ is canonically defined for each finite subset $\Lambda \subset \mathbb{Z}^{d}$ as

$$
\varphi_{\Lambda}=\operatorname{Tr}_{\mathfrak{A}_{\Lambda}}\left(e^{-h_{\Lambda}} \cdot\right)
$$

The potential $\left\{h_{\Lambda}\right\}_{\Lambda \subset \mathbb{Z}^{d}}$ satisfies standard normalization conditions, together with compatibility conditions. In addition, if the state $\varphi$ is consistent with a net $\left\{E_{\Lambda^{\prime}}\right\}_{\Lambda \subset \mathbb{Z}^{d}}$ of conditional expectations as that in Theorem 1, then the potential satisfies some nice commutation relations. For example, if $\Lambda \subset \subset \widehat{\Lambda}$ are bounded regions, then

$$
\begin{aligned}
h_{\widehat{\Lambda}} & =H_{\widehat{\Lambda} \backslash \Lambda}+K_{\bar{\Lambda}}, \\
h_{\widehat{\Lambda} \backslash \Lambda} & =H_{\widehat{\Lambda} \backslash \Lambda}+M_{\partial \Lambda}
\end{aligned}
$$

for selfadjoint operators $H_{\widehat{\Lambda} \backslash \Lambda} \in \mathfrak{A}_{\widehat{\Lambda} \backslash \Lambda}, K_{\bar{\Lambda}} \in \mathfrak{A}_{\bar{\Lambda}}$, and $M_{\partial \Lambda} \in \mathfrak{A}_{\partial \Lambda}$. Further, the above operators satisfy the following commutation relations

$$
\left[H_{\widehat{\Lambda} \backslash \Lambda}, K_{\bar{\Lambda}}\right]=\left[H_{\widehat{\Lambda} \backslash \Lambda}, M_{\partial \Lambda}\right]=0
$$

see [4], Lemma 4.1.

Now we are in position to give the converse of Theorem 1.

Theorem 2 (Theorem 4.2 of [4])

Let be given a locally faithful state $\varphi \in \mathcal{S}(\mathfrak{A})$, together with a net $\left\{E_{\Lambda^{\prime}}\right\}_{\Lambda \in \mathcal{F}}$ of Umegaki conditional expectations satisfying conditions $(i)-(i i i)$ of Theorem 1 .

Then $\varphi$ is a Markov state in the sense of Definition 2.1.

Proof 2 The proof easily follows from (6), (7). Namely, we have for the $\varphi_{\widehat{\Lambda}}$-conditional expectation,

$$
E_{\widehat{\Lambda} \backslash \Lambda}^{\varphi_{\widehat{\Lambda}}}(A)=\left(\operatorname{id}_{\mathfrak{A}_{\widehat{\Lambda} \backslash \Lambda}} \otimes \operatorname{Tr}_{\mathfrak{A}_{\Lambda}}\right)\left(k_{\widehat{\Lambda}, \Lambda}^{*} A k_{\widehat{\Lambda}, \Lambda}\right)
$$

where $k_{\widehat{\Lambda}, \Lambda}$ is the transition operator given by

$$
k_{\widehat{\Lambda}, \Lambda}=e^{-\frac{1}{2} h_{\widehat{\Lambda}}} e^{\frac{1}{2} h_{\widehat{\Lambda} \backslash \Lambda}}
$$

Thus, in our situation we get

$$
k_{\widehat{\Lambda}, \Lambda}=e^{-\frac{1}{2} K_{\bar{\Lambda}}} e^{\frac{1}{2} M_{\partial \Lambda}} \in \mathfrak{A}_{\bar{\Lambda}}
$$

that is $E_{\widehat{\Lambda} \backslash \Lambda}^{\varphi_{\widehat{\Lambda}}}$ acts as the identity on $\mathfrak{A}_{\widehat{\Lambda} \backslash \Lambda}$. 


\section{The relation with statistical mechanics}

We start with a Markov state $\varphi$ on the quasi-local algebra $\mathfrak{A}$. The potential $\left\{h_{\Lambda}\right\}_{\Lambda \subset \mathbb{Z}^{d}}$ associated to $\varphi$ by (5) satisfies nice properties like those given in (6), (7). This allows us to check that, w.r.t. the order $\Lambda \subset \subset \widehat{\Lambda}$, the pointwise-norm limit

$$
\alpha_{t}(a):=\lim _{\Lambda \uparrow \mathbb{Z}^{d}} e^{-i t h_{\Lambda}} a e^{i t h_{\Lambda}}
$$

exists and defines a strongly continuous one-parameter automorphisms group of $\mathfrak{A}$. By constuction, $\varphi$ is automatically a KMS state for $\alpha_{t}$ (i.e. a KMS state at inverse temperature $\beta=-1$ ). In addition, $\varphi$ is faithful, see [4], Section 5.

The disintegration theory of states which are invariant w.r.t. a net of conditional expectations which act locally, was developed in [1]. For such a disintegration, one should start by considering the natural extensions $\epsilon_{\Lambda^{\prime}}^{\varphi}$ of the $E_{\Lambda^{\prime}}^{\varphi}$ to all of $\pi_{\varphi}(\mathfrak{A})^{\prime \prime}$ given by

$$
\epsilon_{\Lambda^{\prime}}^{\varphi}:=\mathcal{E}_{\Lambda^{\prime}} \otimes \operatorname{id}_{\pi_{\varphi}\left(\mathfrak{A}_{\Lambda^{\prime}}\right)^{\prime \prime}}
$$

The projections $\left\{e_{\Lambda^{\prime}}^{\varphi}\right\}_{\Lambda \subset \mathbb{Z}^{d}}$ are then the cyclic projections relative to the ranges $\mathcal{R}\left(\epsilon_{\Lambda^{\prime}}^{\varphi}\right)$ of the $\epsilon_{\Lambda^{\prime}}^{\varphi}$. The orthogonal measure ([19], Section 3) corresponding to such a disintegration is precisely the $\left\{\pi_{\varphi}(\mathfrak{A}),\left\{e_{\Lambda^{\prime}}^{\varphi}\right\}\right\}^{\prime}$-measure, see [1], Theorem 3.2.

In addition, a Markov state $\varphi$ is also a KMS state, then one can use standard results relative to the disintegration of a KMS state into states describing pure phases, see e.g. [9]. The main result is that both disintegrations coincide. Namely, in the situation under consideration we have

$$
\mathfrak{Z}_{\varphi}^{\perp}=\left\{\pi_{\varphi}(\mathfrak{A}),\left\{e_{\Lambda^{\prime}}^{\varphi}\right\}\right\}^{\prime}=\mathfrak{Z}_{\varphi}
$$

where, for $\Lambda$ bounded,

$$
\mathfrak{Z}_{\varphi}^{\perp}:=\bigwedge_{\Lambda \subset \mathbb{Z}^{d}} \pi_{\varphi}\left(\mathfrak{A}_{\Lambda^{\prime}}\right)^{\prime \prime}
$$

is the algebra at infinity, and

$$
\mathfrak{Z}_{\varphi}:=\pi_{\varphi}(\mathfrak{A})^{\prime} \wedge \pi_{\varphi}(\mathfrak{A})^{\prime \prime}
$$

is the centre of the GNS representation of the state $\varphi$.

We collect the main properties of a Markov state in the following 
Theorem 3 (Theorem 5.4 of [4])

Let $\varphi \in \mathcal{S}(\mathfrak{A})$ be a Markov state.

(i) The state $\varphi$ is a $\left\{\alpha_{t}\right\}-K M S$ state, where $\alpha_{t}$ is the automorphisms group given in (8),

(ii) the state $\varphi$ admits a disintegration

$$
\varphi=\int_{\mathcal{S}(\mathfrak{A})} \psi \mu(d \psi)
$$

where the measure $\mu$ is concentrated on the factor states of $\mathfrak{A}$,

(iii) Almost all $\psi$ appearing in (9) are $\left\{\alpha_{t}\right\}-K M S$ states, and Markov states in the sense of Definition 2.1.

To conclude, we shortly describe the possible appearence of the wellknown phenomenon of symmetry breaking. In order to do this, we consider translationally invariant Markov states.

Let $\tau_{x}: \mathfrak{A} \mapsto \mathfrak{A}$ be the shift generated in a natural way on the spin algebra by the translation $x \in \mathbb{Z}^{d}$, and consider a Markov state $\varphi \in \mathcal{S}(\mathfrak{A})$ invariant w.r.t. $\left\{\tau_{x}\right\}_{x \in \mathbb{Z}^{d}}$. One easily verifies that the generalized conditional expectations associated to the restrictions of $\varphi$ satisfy

$$
E_{\widehat{\Lambda} \backslash \Lambda}^{\varphi_{\widehat{\Lambda}}}=\tau_{x}^{-1} \circ E_{\widehat{\Lambda}+x \backslash \Lambda+x}^{\varphi_{\widehat{\Lambda}+x}} \circ \tau_{x}
$$

which yields for the corresponding Umegaki conditional expectations given in Theorem 1,

$$
E_{\Lambda^{\prime}}=\tau_{x}^{-1} \circ E_{\Lambda^{\prime}+x} \circ \tau_{x}
$$

Consider the dual action $\left\{T_{x}\right\}_{x \in \mathbb{Z}^{d}}$ on the state space of $\mathfrak{A}$. It is straightforward to verify that $T_{x}$ maps the set $\mathcal{S}_{E} \subset \mathcal{S}(\mathfrak{A})$ of all $\left\{E_{\Lambda^{\prime}}\right\}$-invariant states into itself, leaving globally stable the set $\partial \mathcal{S}_{E}$ of its extremal states. We easily conclude by the uniqueness of the maximal measure ([7], Theorem II.3.6), that $T_{x}$ preserves the measure $\mu$ given in (9):

$$
\mu \circ T_{x}=\mu \text {. }
$$

We then have a measure-preserving $\mathbb{Z}^{d-a c t i o n} x \mapsto T_{x}$ on the state space $\mathcal{S}(\mathfrak{A})$ of $\mathfrak{A}$. 
Fix our attention on an ergodic component $m$ of the ergodic disintegration

$$
\mu=\int m \nu(d m)
$$

of the measure $\mu .^{2}$ If the standard measure space $(\mathcal{S}(\mathfrak{A}), m)$ is essentially transitive for the action of $\mathbb{Z}^{d}$ (that is when $m$ is concentrated on a single orbit), then the $\mathbb{Z}^{d}$-space $(\mathcal{S}(\mathfrak{A}), m)$ is similar to the $\mathbb{Z}^{d}$-space $\left(\mathbb{Z}^{d} / H, \lambda\right)$ based on the homogeneous space $\mathbb{Z}^{d} / H$, see [17], Theorem 4.12 (see [16] for the original result). Here $H \subset \mathbb{Z}^{d}$ is a subgroup of $\mathbb{Z}^{d}$, and $\lambda$ is a probability measure equivalent to the Haar measure on the Abelian group $\mathbb{Z}^{d} / H$.

In the $\left\{E_{\Lambda^{\prime}}\right\}$-extremal disintegration of a $\left\{\tau_{x}\right\}$-invariant Markov state $\varphi$, states with a smaller symmetry might occur. In our example, the surviving symmetry group is precisely the subgroup $H \subset \mathbb{Z}^{d}$. This is an instance of the phenomenon of symmetry breaking, see e.g. $[8,9,18]$ for quite similar situations and for technical details.

\section{Markov states on non homogeneous chains: general properties}

General properties of quantum Markov states were firstly investigated for one-dimensional models where the order plays a crucial role. Recently, taking into account results contained in $[5,6]$, the emerging structure has been fully understood, see [3]. Here we report the main results relative to quantum Markov states on chains.

We start by considering a totally ordered countable discrete set $I$ containing, possibly a smallest element $j_{-}$and/or a greatest element $j_{+}$. Namely, if $I$ contains neither $j_{-}$, nor $j_{+}$, then $I \sim \mathbb{Z}$. If just $j_{+} \in I$, then $I \sim \mathbb{Z}_{-}$, whereas if only $j_{-} \in I$, then $I \sim \mathbb{Z}_{+}$. Finally, if both $j_{-}$and $j_{+}$belong to $I$, then $I$ is a finite set and the analysis becomes easier. If $I$ is order-isomorphic to $\mathbb{Z}, \mathbb{Z}_{-}$or $\mathbb{Z}_{+}$, we put simbolically $j_{-}$and/or $j_{+}$equal to $-\infty$ and/or $+\infty$ respectively. In such a way, the objects with indices $j_{-}$and $j_{+}$will be missing in the computations. The bounded sets $\Lambda_{k, j}:=[k, j]$ assume a fundamental role in the sequel.

\footnotetext{
${ }^{2}$ The ergodic disintegration of the measure $\mu$ corresponds to the direct-integral disintegration ([21], Theorem 8.21) of the von Neumann algebra $L^{\infty}(\mathcal{S}(\mathfrak{A}), \mu)$ w.r.t. the fixed-point subalgebra relative to the natural $\mathbb{Z}^{d}$-action on $L^{\infty}(\mathcal{S}(\mathfrak{A}), \mu)$.
} 
In this situation, the spin algebra is the quasi-local algebra $\mathfrak{A}$ obtained by the infinite $C^{*}$-tensor product of full matrix algebras $\left\{M^{j}\right\}_{j \in I}$ with possibly different dimensions:

$$
M^{j}:=\mathbb{M}_{k_{j}}(\mathbb{C}) .
$$

Notice that the present situation naturally arises from the previous one when we restrict ourselves to any increasing sequence of bounded regions $\left\{\Gamma_{k}\right\}_{k \in \mathbb{N}}$ of $\mathbb{Z}^{d}$ satisfying

$$
\Gamma_{k+1}=\overline{\Gamma_{k}}
$$

and exhausting all of $\mathbb{Z}^{d}$ :

$$
\bigcup_{k \in \mathbb{N}} \Gamma_{k}=\mathbb{Z}^{d}
$$

We have,

$$
M^{j}=\mathfrak{A}_{\Gamma_{-k} \backslash \Gamma_{-k-1}}
$$

and $I \sim \mathbb{Z}_{-} \cdot{ }^{3}$

We start with a locally faithful state $\varphi$ on the quasi-local algebra $\mathfrak{A}$. Denote

$$
E_{\Lambda_{k, l}}^{\varphi_{\Lambda_{k, l+1}}}: \mathfrak{A}_{\Lambda_{k, l+1}} \mapsto \mathfrak{A}_{\Lambda_{k, l}}
$$

the $\varphi_{\Lambda_{k, l+1}}$-conditional expectation relative to the inclusion $\mathfrak{A}_{\Lambda_{k, l}} \subset \mathfrak{A}_{\Lambda_{k, l+1}}$, $k \leq l$.

Definition 4.1 The locally faithful state $\varphi \in \mathcal{S}(\mathfrak{A})$ is said to be a Markov state on the chain if for every $k, l \in I$ with $k \leq l$, we have

$$
\mathfrak{A}_{\Lambda_{k, l-1}} \subset \operatorname{Fix}\left(E_{\Lambda_{k, l}}^{\varphi_{\Lambda_{k, l+1}}}\right)
$$

Also in this situation, we can find a net of Umegaki conditional expectations which act locally, and leave fixed the Markov state $\varphi$. This can be done by recovering a very explicit structure of the potential associated to $\varphi$.

Theorem 4 (Theorem 5.1 of [3])

Let $\varphi \in \mathcal{S}(\mathfrak{A})$ be locally faithful.

Then the following assertions are equivalent.

(i) $\varphi$ is a Markov state on the chain.

\footnotetext{
${ }^{3}$ According to the previous literature $([5,6])$, we are using the reverse order.
} 
(ii) For each $k \leq l$, the potential $\left\{h_{\Lambda_{k, l}}\right\}$ associated to $\varphi$ by (5), can be recovered by

$$
h_{\Lambda_{k, l}}=H_{k}+\sum_{j=k}^{l-1} H_{j, j+1}+\widehat{H}_{l}
$$

from sequences $\left\{H_{j}\right\}_{j_{-} \leq j \leq j_{+}},\left\{\widehat{H}_{j}\right\}_{j_{-} \leq j \leq j_{+}}$and $\left\{H_{j, j+1}\right\}_{j_{-} \leq j<j_{+}}$of selfadjoint operators localized in $\mathfrak{A}_{\Lambda_{j, j}}$ and $\mathfrak{A}_{\Lambda_{j, j+1}}$ respectively. Such sequences satisfy the commutation relations

$$
\begin{gathered}
{\left[H_{j}, H_{j, j+1}\right]=0} \\
{\left[H_{j, j+1}, \widehat{H}_{j+1}\right]=0,} \\
{\left[H_{j}, \widehat{H}_{j}\right]=0, \quad\left[H_{j, j+1}, H_{j+1, j+2}\right]=0}
\end{gathered}
$$

(iii) For every $k \leq l \leq j$ there exists a sequence of Umegaki conditional expectations $\left\{E_{k, j}\right\}$ defined on the algebras $\left\{\mathfrak{A}_{\Lambda_{k, j+1}}\right\}$. Such a sequence satisfies

$$
\begin{gathered}
E_{k, j}\left(\mathfrak{A}_{\Lambda_{k, j+1}}\right) \subset \mathfrak{A}_{\Lambda_{k, j}}, \\
E_{k, j}\left\lceil\mathfrak{A}_{\Lambda_{k, j-1}}=\mathrm{id}_{\mathfrak{A}_{\Lambda_{k, j-1}}},\right. \\
E_{k, j}\left\lceil_{\mathfrak{A}_{\Lambda_{l, j}}}=E_{l, j}\right.
\end{gathered}
$$

and leaves invariant the state $\varphi$ :

$$
\varphi_{\Lambda_{k, j}} \circ E_{k, j}=\varphi_{\Lambda_{k, j+1}}
$$

The proof of the last theorem parallels those of Theorem 1 and Theorem 2. We refer the reader to [3] for details.

For one-dimensional models, the connection with statistical mechanics is fully clarified. Namely, the Markov property for a locally faithful state $\varphi$ is characterized by the existence of a very explicit nearest neighbour Hamiltonian (13) canonically associated to $\varphi$. Such a potential generates a oneparameter group of automorphisms of the quasi-local algebra $\mathfrak{A}$, admitting $\varphi$ as a KMS-state, see [3], Theorem 5.3.

From $\left\{E_{k, l}\right\}$, we recover a sequence $\left\{\mathcal{E}^{j}\right\}_{j_{-} \leq j<j_{+}}$of transition expectations

$$
\mathcal{E}^{j}: M^{j} \otimes M^{j+1} \mapsto \mathcal{R}\left(\mathcal{E}^{j}\right) \subset M^{j}
$$


Namely,

$$
E_{k, j}=\operatorname{id}_{\mathfrak{A}_{\Lambda_{k, j-1}}} \otimes \mathcal{E}^{j}
$$

where

$$
\mathcal{E}^{j}=E_{k, j}\left\lceil\mathfrak{A}_{\Lambda_{j, j+1}}\right.
$$

It is straightforward to show that such transition expectations satisfy ${ }^{4}$

$$
\mathcal{E}^{j-1}(A \otimes B)=\mathcal{E}^{j-1}\left(A \otimes \mathcal{E}^{j}(B \otimes I)\right) \quad j_{-}<j<j_{+}
$$

Finally, we remark that also the last situation can be fitted into quantum Markov processes on directed sets. Namely, the directed set is precisely $\left\{\Lambda_{k, l}\right\}_{j_{-}<k \leq l<j_{+}}$, with boundary

$$
\partial \Lambda_{k, l}=\Lambda_{k-1, k-1} \cup \Lambda_{l+1, l+1}
$$

\section{The structure of Markov states on chains: a reconstuction theorem}

We start by studying the structure of Markov states on chains. This can be done by using the explicit description of Umegaki conditional expectations on matrix algebras. In such a way, we obtain a disintegration of a Markov state into "elementary Markov states" in a sense we are going to explain.

In order to treat also the most general case of non locally faithful states, only in the present section we adopt the following definition for quantum Markov states on chains.

Definition 5.1 Let $\varphi \in \mathcal{S}(\mathfrak{A})$. The state $\varphi$ is said to be a Markov state if $\varphi$ satisfies condition (iii) of Theorem 4 , for some sequence $\left\{E_{k, l}\right\}_{j_{-} \leq k \leq l \leq j_{+}}$of Umegaki conditional expectations.

As the sequence $\left\{E_{k, l}\right\}$ determines and is determined by a sequence of transition expectations $\left\{\mathcal{E}^{j}\right\}$ as in (14), we speak about the Markov property w.r.t. the sequence $\left\{\mathcal{E}^{j}\right\}$.

Let $\varphi$ be a Markov state on the quasi-local algebra $\mathfrak{A}$ w.r.t. the sequence $\left\{\mathcal{E}^{j}\right\}_{j_{-} \leq j<j_{+}}$of transition expectations. We consider the centre $Z^{j}$, with

\footnotetext{
${ }^{4}$ In the most general case of non locally faithful states considered in the next section, relations (15) could be not satisfied in general.
} 
spectrum $\Omega_{j}$, of the range $\mathcal{R}\left(\mathcal{E}^{j}\right)$ of $\mathcal{E}^{j}$, together with the generating family $\left\{P_{\omega_{j}}^{j}\right\}_{\omega_{j} \in \Omega_{j}}$ of atomic projections. We set

$$
B^{j}:=\oplus_{\omega_{j} \in \Omega_{j}} P_{\omega_{j}}^{j} M^{j} P_{\omega_{j}}^{j}
$$

and define

$$
\mathfrak{B}:=\left(\otimes_{j_{-} \leq j<j_{+}} B^{j}\right) \otimes M^{j_{+}}
$$

Then we obtain in a canonical way, a conditional expectation

$$
E: \mathfrak{A} \mapsto \mathfrak{B}
$$

defined to be the (infinite) tensor product of the following conditional expectations

$$
a \in M^{j} \mapsto \sum_{\omega_{j} \in \Omega_{j}} P_{\omega_{j}}^{j} a P_{\omega_{j}}^{j}
$$

together with the identity map on $M^{j_{+}}$. The reduced algebra

$$
M_{P_{\omega_{j}}^{j}}^{j} \equiv P_{\omega_{j}}^{j} M^{j} P_{\omega_{j}}^{j}
$$

can be written as

$$
M_{P_{\omega_{j}}^{j}}^{j}=N_{\omega_{j}}^{j} \otimes \bar{N}_{\omega_{j}}^{j}
$$

with $N_{\omega_{j}}^{j}$ and $\bar{N}_{\omega_{j}}^{j}$ all finite-dimensional factors. Again, the states $\phi_{\omega_{j}}^{j}$ on $\bar{N}_{\omega_{j}}^{j} \otimes M^{j+1}$ are uniquely recovered by the transition expectation $\mathcal{E}^{j}$ according to Formula (1).

Following [6], we can recover

a classical Markov process on the compact space

$$
\Omega:=\prod_{j_{-} \leq j<j_{+}} \Omega_{j}
$$

whose law $\mu$ is uniquely determined by the initial distribution and transition probabilities given respectively by

$$
\begin{gathered}
\pi_{\omega_{j_{-}}}^{j_{-}}:=\varphi\left(\iota_{\Lambda_{j_{-}, j_{-}}}\left(P_{\omega_{j_{-}}}^{j_{-}}\right)\right) \\
\pi_{\omega_{j}, \omega_{j+1}}^{j}:=\phi_{\omega_{j}}^{j}\left(I \otimes P_{\omega_{j+1}}^{j+1}\right)
\end{gathered}
$$

The measure space $(\Omega, \mu)$ is obtained as the projective limit of compatible measure spaces $\left\{\left(\Omega_{\Lambda}, \mu_{\Lambda}\right)\right\}_{\Lambda \subset I}$, we denote by $q_{\Lambda}: \Omega \mapsto \Omega_{\Lambda}$ the canonical projection of $\Omega$ onto $\Omega_{\Lambda}$. 
Let $\Omega_{0} \subset \Omega$ be the set consisting of those $\omega \in \Omega$ such that all $\pi_{q_{\Lambda_{j, j}}(\omega)}^{j}$ together with $\pi_{q_{\Lambda_{j, j}}^{j}(\omega), q_{\Lambda_{j+1, j+1}}(\omega)}$ are nonvanishing. the set $\Omega_{0}$ is a measurable set of full $\mu$-measure.

Consider, for each $\omega \in \Omega$, the (infinite) tensor product $\mathfrak{B}_{\omega}$ given by

$$
\begin{gathered}
\mathfrak{B}_{\omega}:=\left(\otimes_{j_{-} \leq j<j_{+}} M_{P_{\omega_{j}}^{j}}^{j}\right) \otimes M^{j_{+}} \\
\equiv N_{\omega_{j_{-}}^{j_{-}}}^{j_{j_{-}}} \otimes\left(\otimes_{j_{-} \leq j<j_{+}-1}\left(\bar{N}_{\omega_{j}}^{j} \otimes N_{\omega_{j+1}}^{j+1}\right)\right) \otimes\left(\bar{N}_{\omega_{j_{+}-1}^{j_{+}-1}} \otimes M^{j_{+}}\right)
\end{gathered}
$$

A completely positive identity-preserving map $E_{\omega}: \mathfrak{A} \mapsto \mathfrak{B}_{\omega}$ is uniquely defined as the (infinite) tensor product of the maps

$$
a \in M^{j} \mapsto P_{q_{\Lambda_{j, j}}(\omega)}^{j} a P_{q_{\Lambda_{j, j}}^{j}(\omega)}^{j}
$$

together with the identity map on $M^{j_{+}}$. We have trivially

$$
E_{\omega} \circ E=E_{\omega}
$$

where $E$ is obtained by the (infinite) tensor product of the maps given in (17).

Denoting (with an abuse of notation) by $\omega_{j}$ the canonical projection $q_{\Lambda_{j, j}}(\omega)$ of $\omega$ in $\Omega_{j}$, we further recover for $\omega \in \Omega_{0}$

states $\psi_{\omega}$ on $\mathfrak{B}_{\omega}$ given by

$$
\psi_{\omega}:=\eta_{q_{\Lambda_{j_{-}, j_{-}}}}^{j_{-}} \otimes\left(\otimes_{j_{-} \leq j<j_{+}-1} \eta_{q_{\Lambda_{j, j}}(\omega), q_{\Lambda_{j+1, j+1}}(\omega)}^{j}\right) \otimes \eta_{q_{\Lambda_{j_{+}-1, j_{+}-1}}^{j_{+}-1}(\omega)}
$$

determined by the initial distribution, which is the state on $N_{\omega_{j_{-}}}^{j_{-}}$given by

$$
\eta_{\omega_{j_{-}}}^{j_{-}}(a):=\frac{\varphi\left(\iota_{\Lambda_{j_{-}, j_{-}}}\left(P_{\omega_{j_{-}}}^{j_{-}}(a \otimes I) P_{\omega_{j_{-}}}^{j_{-}}\right)\right)}{\pi_{\omega_{j_{-}}}^{j_{-}}}
$$

by the states $\eta_{\omega_{j}, \omega_{j+1}}^{j}$ on $\bar{N}_{\omega_{j}}^{j} \otimes N_{\omega_{j+1}}^{j+1}$, given by

$$
\eta_{\omega_{j}, \omega_{j+1}}^{j}(\bar{a} \otimes b):=\frac{\phi_{\omega_{j}}^{j}\left(\bar{a} \otimes P_{\omega_{j+1}}^{j+1}(b \otimes I) P_{\omega_{j+1}}^{j+1}\right)}{\pi_{\omega_{j}, \omega_{j+1}}^{j}}
$$

and by the final distribution which is the state on $\bar{N}_{\omega_{j_{+}-1}}^{j_{+}-1} \otimes M^{j_{+}}$given by

$$
\eta_{\omega_{j_{+}-1}}^{j_{+}-1}(\bar{a} \otimes B):=\phi_{\omega_{j_{+}-1}}^{j_{+-1}}(\bar{a} \otimes B)
$$


Finally, we recover

a sequence $\left\{\mathcal{E}_{\omega}{ }^{j}\right\}_{j_{-} \leq j<j_{+}}$of conditional expectations

$$
\begin{aligned}
\mathcal{E}_{\omega}{ }^{j}: & M_{P_{\omega_{j}}^{j}}^{j} \otimes M_{P_{\omega_{j+1}}^{j+1}}^{j+1} \mapsto M_{P_{\omega_{j}}^{j}}^{j}, \\
\mathcal{E}_{\omega}^{j_{+}-1}: & M_{P_{\omega_{j_{+}-1}}^{j_{+}-1}}^{j-1} \otimes M^{j_{+}} \mapsto M_{P_{\omega_{j_{+}-1}}^{j_{+}-1}}^{j_{+}}
\end{aligned}
$$

given by

$$
\begin{aligned}
& \mathcal{E}_{\omega}{ }^{j}((a \otimes \bar{a}) \otimes(b \otimes \bar{b}))=\eta_{\omega_{j}, \omega_{j+1}}^{j}(\bar{a} \otimes b) \eta_{\omega_{j+1}, \omega_{j+2}}^{j+1}(\bar{b} \otimes I) a \otimes I, \\
& \mathcal{E}_{\omega}{ }^{j_{+}-1}((a \otimes \bar{a}) \otimes B)=\quad \eta_{\omega_{j_{+}-1}^{j_{+}-1}}(\bar{a} \otimes B) a \otimes I
\end{aligned}
$$

It is easy to verify that the states $\psi_{\omega}, \omega \in \Omega_{0}$, are Markov states on $\mathfrak{B}_{\omega}$ w.r.t $\left\{\mathcal{E}_{\omega}{ }^{j}\right\}_{j_{-} \leq j<j_{+}}$given by (28), respectively. Further, the map

$$
\omega \in \Omega_{0} \mapsto \psi_{\omega} \circ E_{\omega} \in \mathcal{S}(\mathfrak{A})
$$

is $\sigma\left(\mathfrak{A}^{*}, \mathfrak{A}\right)$-measurable.

We are ready to report the announced result concerning the disintegration of a Markov state into elementary Markov states which are minimal in the sense that the ranges of the associated transition expectations have a trivial centre.

Theorem 5 (Theorem 3.2 of [3])

Let $\varphi$ be a Markov state on the quasi-local algebra $\mathfrak{A}$ w.r.t. the sequence $\left\{\mathcal{E}_{j}\right\}_{j_{-} \leq j<j_{+}}$of transition expectations.

Define the set $\Omega$ by (19); the probability measure $\mu$ on $\Omega$, by (20); the quasi-local algebra $\mathfrak{B}_{\omega}$ by (21), the map $E_{\omega}$ by the projections (22); the state $\psi_{\omega}$ on $\mathfrak{B}_{\omega}$ by (24).

Then $\varphi$ admits a disintegration

$$
\varphi=\int_{\Omega} \varphi_{\omega} \mu(d \omega)
$$

where $\omega \in \Omega \mapsto \varphi_{\omega} \in \mathcal{S}(\mathfrak{A})$ is a $\sigma\left(\mathfrak{A}^{*}, \mathfrak{A}\right)$-measurable map satisfying, for $\mu$-almost all $\omega \in \Omega$,

$$
\varphi_{\omega}=\psi_{\omega} \circ E_{\omega}
$$


The proof of the above theorem relies on standard techniques of the theory of direct-integral decomposition of representations of $C^{*}$-algebras. Such a proof can be found in [3].

Notice that the GNS representations $\pi_{\varphi_{\omega}}$ of $\varphi_{\omega}$ give rise to von Neumann factors. The proof of the last assertion follows from [8], Theorem 2.6.10. Further, the disintegration (29), even if it is made of factor states, does not correspond to the central disintegration given in Theorem 3.

The following theorem is the converse of Theorem 5 and can be also regarded as a reconstruction result for quantum Markov states on chains.

Consider for $j_{-} \leq j<j_{+}$, a sequence $Z^{j}$ of commutative subalgebras of $M^{j}$ with spectra $\Omega_{j}$ and generators $\left\{P_{\omega_{j}}^{j}\right\}_{\omega_{j} \in \Omega_{j}}$; a Markov process on the product space

$$
\Omega:=\prod_{j_{-} \leq j<j_{+}} \Omega_{j}
$$

with law $\mu$ determined, for $\omega_{j} \in \Omega_{j}, \omega_{j+1} \in \Omega_{j+1}$, by all marginal distributions $\pi_{\omega_{j}}^{j}$, and all transition probabilities $\pi_{\omega_{j}, \omega_{j+1}}^{j}$.

For $\omega_{j} \in \Omega_{j}$ such that $\pi_{\omega_{j}}^{j}>0$, fix a splitting as (18)

$$
M_{P_{\omega_{j}}^{j}}^{j}=N_{\omega_{j}}^{j} \otimes \bar{N}_{\omega_{j}}^{j}
$$

by finite-dimensional factors.

For $\omega_{j_{-}} \in \Omega_{j_{-}}$such that $\pi_{\omega_{j_{-}}}^{j_{-}}>0$, choose a initial distribution $\eta_{\omega_{j_{-}}}^{j_{-}}$on $N_{\omega_{j_{-}}}^{j_{-}}$.

For each pair $\left(\omega_{j}, \omega_{j+1}\right) \in \Omega_{j} \times \Omega_{j+1}$ such that $\pi_{\omega_{j}, \omega_{j+1}}^{j}>0$, consider a state $\eta_{\omega_{j}, \omega_{j+1}}^{j}$ on $\bar{N}_{\omega_{j}}^{j} \otimes N_{\omega_{j+1}}^{j+1}$.

For $\omega_{j_{+}-1} \in \Omega_{j_{+}-1}$ such that $\pi_{\omega_{j_{+}-1}}^{j_{+}-1}>0$, consider a final distribution $\eta_{\omega_{j_{+}-1}}^{j_{+}-1}$ on $N_{\omega_{j_{+}-1}^{j_{+}-1}}^{j_{-}} \otimes M^{j_{+}}$.

Then, on the measurable set $\Omega_{0}$ of full $\mu$-measure consisting of sequences $\omega$ such that all the $\pi_{q_{\Lambda_{j, j}}(\omega)}^{j}$, and $\pi_{q_{\Lambda_{j, j}}(\omega), q_{\Lambda_{j+1, j+1}}(\omega)}$ are nonvanishing, the state $\psi_{\omega}$ in (24) is a well-defined Markov state on the quasi-local algebra $\mathfrak{B}_{\omega}$ given in (21) w.r.t. the sequence $\left\{\mathcal{E}_{\omega}{ }^{j}\right\}_{j_{-} \leq j<j_{+}}$of transition expectations (28). Finally, defining $E_{\omega}: \mathfrak{A} \mapsto \mathfrak{B}_{\omega}$ by (22), the map

$$
\omega \in \Omega_{0} \mapsto \psi_{\omega} \circ E_{\omega}=: \varphi_{\omega} \in \mathcal{S}(\mathfrak{A})
$$

is $\sigma\left(\mathfrak{A}^{*}, \mathfrak{A}\right)$-measurable. 
Theorem 6 (Theorem 4.1 of [3])

Let $\varphi_{\omega}$ be the measurable field on $\mathcal{S}(\mathfrak{A})$ given by (30).

Then the state $\varphi$ on $\mathfrak{A}$ given by

$$
\varphi:=\int_{\Omega_{0}} \varphi_{\omega} \mu(d \omega)
$$

is a Markov state w.r.t. any sequence $\left\{\mathcal{E}^{j}\right\}_{j_{-} \leq j<j_{+}}$of transition expectations with $\mathcal{Z}\left(\mathcal{R}\left(\mathcal{E}^{j}\right)\right)=Z^{j}$, determined according to (1), by states $\phi_{\omega_{j}}^{j}$ satisfying, for each $j_{-} \leq j<j_{+}$and $\omega_{j} \in \Omega_{j}$,

$$
\begin{array}{cc}
\pi_{\omega_{j}}^{j} \phi_{\omega_{j}}^{j} & \left(\bar{a} \otimes P_{\omega_{j+1}}^{j+1}(b \otimes \bar{b}) P_{\omega_{j+1}}^{j+1}\right) \\
= & \sum_{\omega_{j+2}} \pi_{\omega_{j}}^{j} \pi_{\omega_{j}, \omega_{j+1}}^{j} \pi_{\omega_{j+1}, \omega_{j+2}}^{j+1} \eta_{\omega_{j}, \omega_{j+1}}^{j}(\bar{a} \otimes b) \eta_{\omega_{j+1}, \omega_{j+2}}^{j+1}(\bar{b} \otimes I), \\
\pi_{\omega_{j_{+}-2}}^{j_{+}-2} & \phi_{\omega_{j_{+}-2}}^{j_{+}-2}\left(\bar{a} \otimes P_{\omega_{j_{+}-1}}^{j_{+}-1}(b \otimes \bar{b}) P_{\omega_{j_{+}-1}}^{j_{+}-1}\right) \\
= & \pi_{\omega_{j_{+}-2}}^{j_{+}-2} \pi_{\omega_{j_{+}-2} \omega_{j_{+}-1}}^{j_{+}-2} \eta_{\omega_{j_{+}-2}, \omega_{j_{+}-1}}^{j_{+}-2}(\bar{a} \otimes b) \eta_{\omega_{j_{+}-1}}^{j_{+}-1}(\bar{b} \otimes I), \\
\pi_{\omega_{j_{+}-1}}^{j_{+}-1} & \phi_{\omega_{j_{+}-1}}^{j_{+}-1}(\bar{a} \otimes B) \\
= & \pi_{\omega_{j_{+}-1}}^{j_{+}-1} \eta_{\omega_{j_{+}-1}}^{j_{+}-1}(\bar{a} \otimes B)
\end{array}
$$

The proof of the last theorem consists in showing that the state $\varphi$ is a Markov state w.r.t. any sequence of transition expectations $\left\{\mathcal{E}^{j}\right\}$ constucted by (1), taking into account (31). The reader is referred to [3] for the complete proof.

\section{Quantum Markov states on general quasi- local algebras}

The investigation of the quantum Markov property for multi-dimensional spin systems suggests us the natural definition for Markov states on general quasi-local algebras.

A quasi-local algebra ([8], Definition 2.6.3) is a $C^{*}$-algebra $\mathfrak{A}$ obtained by the $C^{*}$-inductive limit of a net $\left\{\mathfrak{A}_{\alpha}\right\}_{\alpha \in A}$ of $C^{*}$-subalgebras with the same identity. The directed set $A$ has also an orthogonality relation $\perp$ such that

$$
\left[\mathfrak{A}_{\alpha}, \mathfrak{A}_{\beta}\right]=\{0\}, \quad \alpha \perp \beta
$$

For each $\alpha \in A$, one can define

$$
\mathfrak{A}_{\alpha^{\prime}}:=\overline{\bigvee_{\beta \perp \alpha} \mathfrak{A}_{\beta}}
$$


where the bar denotes the uniform closure.

In the previous situations, $A=\left\{\Lambda \subset \mathbb{Z}^{d}, \Lambda\right.$ bounded $\}$ and $\Lambda_{1} \perp \Lambda_{2}$ if $\Lambda_{1} \cap \Lambda_{2}=\emptyset$.

This general situation covers also cases arising from Quantum Field Theory where the $\alpha$ consist of bounded regions of physical space-time, and the orthogonality relation $\perp$ describes Einstein causality, see e.g. [13]. Further, the commutation relations (32) can be suitably replaced in order to include Fermion algebras, or field algebras with different commutation relations.

We are ready to give the definition of the quantum Markov property for the general situation of quasi-local algebras. ${ }^{5}$

Let $\mathfrak{A}$ be a quasi-local algebra together with the local filtration $\left\{\mathfrak{A}_{\alpha}\right\}_{\alpha \in A}$ as above. Suppose that for each $\alpha \in A$, another index $\bar{\alpha} \in A$ is assigned with $\alpha \prec \bar{\alpha}$.

Definition 6.1 A state $\varphi \in \mathcal{S}(\mathfrak{A})$ is said to be a Markov state if there exists a filtration ${ }^{6}\left\{\mathfrak{B}_{\alpha^{\prime}}\right\}_{\alpha \in A}$ of $C^{*}$-subalgebras of $\mathfrak{A}$ such that

$$
\mathfrak{A}_{\bar{\alpha}^{\prime}} \subset \mathfrak{B}_{\alpha^{\prime}} \subset \mathfrak{A}_{\alpha^{\prime}}
$$

together with a projective net $\left\{E_{\alpha^{\prime}}\right\}_{\alpha \in A}$ of Umegaki conditional expectations such that

(i) $E_{\alpha^{\prime}}: \mathfrak{A} \mapsto \mathfrak{B}_{\alpha^{\prime}}$,

(ii) $\varphi \circ E_{\alpha^{\prime}}=\varphi$.

We conclude by noticing that, in order to recover the expected filtration $\left\{\mathfrak{B}_{\alpha^{\prime}}, E_{\alpha^{\prime}}\right\}_{\alpha \in A}$ by more manageable properties, we need additional conditions on the quasi-local algebra $\mathfrak{A}$, as well as on the state $\varphi$ under consideration.

\section{Acknowledgements}

The authors would like to thank V. Liebscher for many valuable discussions.

\footnotetext{
${ }^{5}$ We are indebted to V. Liebscher for fruitful suggestions about this point.

${ }^{6}$ This means that $\alpha \prec \beta \Rightarrow \mathfrak{B}_{\beta^{\prime}} \subset \mathfrak{B}_{\alpha^{\prime}}$.
} 


\section{Riferimenti bibliografici}

[1] Accardi L. Local perturbations of conditional expectations, J. Math. Anal. Appl. 72 (1979), 34-68.

[2] Accardi L., Cecchini C. Conditional expectations in von Neumann algebras and a theorem of Takesaky, J. Funct. Anal. 45 (1982), 245-273.

[3] Accardi L., Fidaleo F. Non homogeneous Markov states and quantum Markov fields, Volterra Preprint 462 (2001).

[4] Accardi L., Fidaleo F. On the structure of quantum Markov fields, Volterra Preprint 486 (2001).

[5] Accardi L., Frigerio A. Markovian cocycles, Proc. R. Ir. Acad. 83 (1983), 251-263.

[6] Accardi L., Liebscher V. Markovian KMS states for one dimensional spin chains, Infin. Dimens. Anal. Quantum Probab. Relat. Top. 2 (1999), 645-661.

[7] Alfsen E. M. Compact convex sets and boundary integral, Springer, Berlin-Heidelberg-New York, 1971.

[8] Bratteli O., Robinson D. W. Operator algebras and quantum statistical mechanics I, Springer, Berlin-Heidelberg-New York, 1981.

[9] Bratteli O., Robinson D. W. Operator algebras and quantum statistical mechanics II, Springer, Berlin-Heidelberg-New York, 1981.

[10] Dobrushin R. L. The description of a random field by means of conditional probabilities and conditions of its regularity, Theory Probab. Appl. 13 (1968), 197-224.

[11] Dobrushin R. L. Gibbsian random fields for lattice systems with pairwaise interactions, Funct. Anal. Appl. 2 (1968), 292-301.

[12] Dobrushin R. L. The problem of uniqueness of a Gibbsian random field and the problem of phase transitions, Funct. Anal. Appl. 2 (1968), 302312 . 
[13] Haag R., Kastler D. An algebraic approach to quantum field theory, J. Math. Phys. 5 (1964), 848-861.

[14] Havet J.-F. Espérance conditionelle minimale, J. Operator Theory 24 (1990), 33-55.

[15] Kümmerer B. A non-commutative ergodic theorem, Invent. Math. 46 (1978), 139-145.

[16] Mackey G. W. Ergodic theory and virtual groups, Math. Ann. 166 (1966), 187-207.

[17] Ramsay A. Virtual groups and group actions, Adv. Math. 6 (1971), $253-322$.

[18] Ruelle D. Statistical mechanics, Benjamin, Amsterdam-New York 1969.

[19] Sakai S. $C^{*}$-algebras and $W^{*}$-algebras, Springer, Berlin-Heidelberg-New York 1971.

[20] Stratila S. Modular theory in operator algebras, Abacus Press, Tunbridge Wells, Kent 1981.

[21] Takesaki M. Theory of operator algebras I, Springer, Berlin-HeidelbergNew York 1979.

[22] Tsukada M. Strong convergence of martingales in von Neumann algebras, Proc. Amer. Math. Soc. 88 (1983), 537-540. 